\title{
Endoftalmites bacterianas com culturas positivas: uma revisão de 6 anos
}

\author{
Culture proven bacterial endophthalmitis: a 6-year review
}

\author{
Paulo José Martins Bispo ${ }^{1}$ \\ Gustavo Barreto de Melo ${ }^{2}$ \\ Pedro Alves d'Azevedo ${ }^{3}$ \\ Ana Luisa Höfling-Lima ${ }^{4}$ \\ Maria Cecília Zorat $\mathbf{Y u}^{5}$ \\ Antonio Carlos Campos Pignatari ${ }^{6}$
}

Trabalho realizado no Departamento de Oftalmologia da Universidade Federal de São Paulo - UNIFESP - São Paulo (SP) - Brasil.

Pós-graduando (nível mestrado) do Laboratório Especial de Microbiologia Clínica - LEMC, Disciplina de Infectologia, Departamento de Medicina, Universidade Federal de São Paulo - UNIFESP - São Paulo (SP) Brasil.

${ }^{2}$ Médico Residente do Serviço de Oftalmologia do Hospital São Paulo, Departamento de Oftalmologia UNIFESP - São Paulo (SP) - Brasil.

3 Pós-doutorado em Doenças Infecciosas e Parasitárias pela UNIFESP- São Paulo (SP) - Brasil. Professor do Departamento de Microbiologia e Parasitologia da Fundação Faculdade Federal de Ciências Médicas de Porto Alegre - FFFCMPA - Porto Alegre (RS) - Brasil

${ }^{4}$ Professora Titular e Chefe do Laboratório de Microbiologia do Departamento de Oftalmologia da UNIFESP São Paulo (SP) - Brasil.

${ }^{5}$ Microbiologista, Laboratório de Microbiologia Ocular do Departamento de Oftalmologia - UNIFESP - São Paulo (SP) - Brasil.

${ }^{6}$ Professor Titular da Disciplina de Infectologia do Departamento de Medicina e Diretor do Laboratório Especial de Microbiologia Clínica da UNIFESP - São Paulo (SP) - Brasil.

Endereço para correspondência: Antonio Carlos Campos Pignatari. Rua Leandro Dupret, 188 - São Paulo (SP) CEP 04025-010.

E-mail: pignatari@terra.com.br

Recebido para publicação em 26.02.2007

Ultima versão recebida em 03.06.2008

Aprovação em 22.06.2008

\section{RESUMO}

Objetivo: Determinar a distribuição dos microrganismos isolados de pacientes com endoftalmite bacteriana e sua sensibilidade a antimicrobianos. Métodos: Foram analisados retrospectivamente os dados clínicos e microbiológicos dos pacientes com hipótese diagnóstica de endoftalmite e cultura bacteriana positiva, atendidos no Departamento de Oftalmologia daUNIFESP de $1^{\circ}$ de janeiro de 2000 a 31 de dezembro de 2005 . Resultados: De 451 pacientes, $153(33,9 \%)$ apresentaram cultura bacteriana positiva. Foram isolados 155 microrganismos, sendo 79,35\% gram-positivos e 20,65\% gram-negativos. Os Staphylococcus coagulase-negativos ( $\mathrm{SCoN})(41,94 \%)$ foram os mais freqüentemente isolados. A sensibilidade aos antimicrobianos entre os gram-negativos foi: amicacina $87,10 \%$, tobramicina $80,65 \%$, ciprofloxacina $96,67 \%$, levofloxacina, gatifloxacina e moxifloxacina $100 \%$, ceftazidima $85 \%$, e gentamicina $80,65 \%$. A sensibilidade à vancomicina entre os gram-positivos foi de $100 \%$. S. aureus e SCoN apresentaram $83,33 \%$ de sensibilidade à oxacilina, $89,61 \%$ à ciprofloxacina e $100 \%$ à gatifloxacina e moxifloxacina. A forma de aquisição predominante foi a pós-operatória (60,65\%). Conclusão: Observamos baixa sensibilidade da cultura para o diagnóstico etiológico das endoftalmites. Uma terapia antimicrobiana ou profilaxia empírica deve ser ativa contra os microrganismos gram-positivos, particularmente contra estafilococos. Estudos de vigilância de resistência bacteriana são importantes para adequação desses esquemas.

Descritores: Infecções oculares bacterianas; Endoftalmite/etiologia; Endoftalmite/cirurgia; Humor aquoso/microbiologia; Resistência microbiana a medicamentos; Testes de sensibilidade microbiana

\section{INTRODUÇÃO}

Endoftalmite bacteriana é uma inflamação intra-ocular decorrente da introdução de um agente bacteriano no segmento posterior do olho. É uma condição rara, mas potencialmente destrutiva para o olho, que gera dano irreversível à delicada camada de células fotorreceptoras da retina, e mesmo com intervenção terapêutica e cirúrgica, freqüentemente resulta em perda parcial ou completa da visão após poucos dias de inoculação ${ }^{(1-2)}$. Os agentes infecciosos podem atingir a porção posterior do olho por três vias: I após cirurgias intra-oculares (pós-operatória), II - após um trauma penetrante no globo ocular (pós-traumática) e III - através de via hematogênica, partindo de um sítio anatômico distante ${ }^{(3)}$. Outra classificação mais ampla considera apenas como endógena ou exógena. A forma exógena ocorre após cirurgia intra-ocular, trauma penetrante, úlcera corneana ou através de quebra de alguma barreira periocular pela qual penetram microrganismos 
infectantes. Comumente, a forma exógena é subseqüente à cirurgia de catarata e, na grande maioria dos casos, é causada por microrganismos provenientes da microbiota ocular que penetram o olho após o procedimento cirúrgico ${ }^{(4)}$. Essas infecções são incomuns, com uma incidência que varia de 0,05 a $0,32 \%^{(1,5-6)}$. Na endoftalmite pós-operatória aguda, os principais microrganismos causadores são Staphylococcus coagulase-negativos (SCoN), Staphylococcus aureus, Streptococcus grupo viridans e, em menor proporção, outros cocos grampositivos e bacilos gram-negativos. Na endoftalmite pós-operatória tardia, os patógenos envolvidos são de baixa virulência, como Propionibacterium acnes, algumas espécies de estreptococos e fungos ${ }^{(7-10)}$. Na endoftalmite pós-traumática, podem ser isolados os mesmos microrganismos causadores de endoftalmite pós-operatória e alguns microrganismos ambientais $^{(11-12)}$. A endoftalmite endógena ocorre em menor proporção que a exógena, correspondendo de 2 a $6 \%$ dos casos de endoftalmite ${ }^{(1,13)}$. Podem ser agentes causadores de endoftalmite endógena S. aureus, Streptococcus pneumoniae, Streptococcus agalactiae, Clostridium perfringens, Moraxella spp., Neisseria meningitidis, Escherichia coli, Klebsiella spp., Serratia marcescens, e Candida albicans ${ }^{(14-21)}$.

$\mathrm{O}$ tratamento eficaz das endoftalmites inclui uma identificação correta e precoce do agente etiológico através da cultura do humor vítreo e/ou aquoso para uma pronta instituição da antibioticoterapia. A escolha do agente antimicrobiano é feita inicialmente de forma empírica. O antimicrobiano de escolha deve possuir atividade contra os patógenos mais relevantes e possuir um amplo espectro de ação. É importante, tanto para o tratamento quanto para a profilaxia, o reconhecimento dos principais microrganismos causadores de endoftalmite com avaliação da emergência de cepas resistentes aos principais antibióticos utilizados em oftalmologia.

\section{MÉTODOS}

Foi realizada análise retrospectiva dos dados clínicos e microbiológicos dos pacientes que apresentaram hipótese diagnóstica de endoftalmite e cultura bacteriana positiva de humor vítreo, aquoso e outros materiais (agulha, bolha, fragmento de córnea, íris, lente intra-ocular e saco capsular), atendidos durante o período de $1^{\circ}$ de janeiro de 2000 a 31 de dezembro de 2005 no Departamento de Oftalmologia da Universidade Federal de São Paulo. Os microrganismos isolados foram identificados pelas características morfo-tintoriais e provas bioquímicas de rotina ${ }^{(22)}$. Os antimicrobianos amicacina, tobramicina, cefazolina, ceftazidima, ciprofloxacina, levofloxacina, gatifloxacina, moxifloxacina, eritromicina, gentamicina, vancomicina, cloranfenicol, oxacilina e penicilina foram testados pelo método de disco difusão de acordo com as recomendações do Clinical Laboratory Standards Institute (CLSI, antigo NCCLS, Filadélfia, EUA) e determinação das concentrações inibitórias mínimas por Etest ${ }^{\circledR}$ (AB Biodisk, Solna, Suécia).

\section{RESULTADOS}

Durante o período do estudo, 451 pacientes foram atendidos com suspeita clínica de endoftalmite e 619 amostras de humor vítreo/aquoso ou outros materiais foram coletados. Desses pacientes, $153(33,9 \%)$ tiveram cultura bacteriana positiva. Das 619 amostras coletadas, 180 apresentaram cultura bacteriana positiva $(29,1 \%)$ e 529 tiveram pedido de bacterioscopia com microscopia positiva para bactéria em $158(29,9 \%)$ casos. Um total de 155 microrganismos foi isolado, já que alguns pacientes apresentaram infecção intra-ocular polimicrobiana. Dos casos de endoftalmites positivos por cultura, em $50,56 \%$ o material colhido foi humor vítreo por punção em 50,56\%, 27,22\% do humor aquoso, $15,56 \%$ do humor vítreo por vitrectomia e $6,67 \%$ foram coletados outros espécimes clínicos.

Os microrganismos gram-positivos representaram o maior número de isolados $79,35 \%$, e os gram-negativos corresponderam a $20,65 \%$. Entre os cocos gram-positivos, os microrganismos mais comumente isolados foram SCoN 41,94\% (65/155), Streptococcus grupo viridans $14,19 \%$ (22/155), S. aureus $8,39 \%$ (13/155) e $S$. pneumoniae 7,10\% (11/155). Os bacilos gramnegativos isolados com maior freqüência foram Pseudomonas sp 4,52\% (7/155) e Haemophilus sp 4,52\% (7/155) (Tabela 1).

Quanto à via de aquisição da infecção, a categoria predominante foi a pós-operatória $(60,6 \%)$, seguida da pós-traumática $(16,1 \%)$ e endógena $(2,6 \%)$. Nos demais casos de endoftalmite $(20,6 \%)$, a via de aquisição não pôde ser classificada. Tanto nas endoftalmites classificadas como pós-operatórias e pós-traumáticas, foi notada uma ampla distribuição dos microrganismos causadores, sendo os SCoN os principais microrganismos isolados, seguidos dos Streptococcus grupo viridans. Quatro casos foram classificados como sendo de origem endógena. Em dois deles, foi isolado $S$. aureus. SCoN foi isolado em um caso e E. coli em outro caso (Tabela 2).

A sensibilidade aos antimicrobianos dos principais microrganismos isolados de endoftalmite no período do estudo é apresentada na tabela 3. Entre os bacilos gram-negativos isolados e testados aos aminoglicosídeos amicacina e tobramicina, a sensibilidade foi de $87,10 \%$ e $80,65 \%$, respectivamente. Em relação às quinolonas, os bacilos gram-negativos apresentaram $100 \%$ de sensibilidade à ciprofloxacina, levofloxacina, gatifloxacina e moxifloxacina, com exceção de $P$. aeruginosa, que apresentou sensibilidade diminuída à ciprofloxacina $(83,33 \%)$. A sensibilidade à ceftazidima foi de $85 \%$ e à gentamicina de $80,65 \%$. Entre os cocos gram-positivos, $100 \%$ dos isolados testados frente à vancomicina foram sensíveis. Os estafilococos $(S$. aureus e $\mathrm{SCoN}$ ), apresentaram $83,33 \%$ de sensibilidade à oxacilina, $89,61 \%$ à ciprofloxacina e $100 \%$ à gatifloxacina e à moxifloxacina. A penicilina apresentou $100 \%$ de atividade contra os isolados de $S$. pneumoniae e $76,4 \%$ contra Streptococcus grupo viridans. Streptococcus pneumoniae e Streptococcus grupo viridans apresentaram, respectivamente, $54,55 \%$ e $45,45 \%$ de sensibilidade à ciprofloxacina, $80 \%$ e $75 \%$ à gatifloxacina e $100 \%$ e $91,67 \%$ à moxifloxacina. 
Tabela 1. Microrganismos isolados de pacientes com diagnóstico clínico de endoftalmite (2000-2005)

\begin{tabular}{|c|c|c|}
\hline Microrganismos isolados & Porcentagem & Número de isolados $(n=155)$ \\
\hline Staphylococcus coagulase negativa & $41,94 \%$ & 65 \\
\hline Streptococcus grupo viridans & $14,19 \%$ & 22 \\
\hline Staphylococcus aureus & $8,39 \%$ & 13 \\
\hline Streptococcus pneumoniae & $7,10 \%$ & 11 \\
\hline Pseudomonas $\mathrm{sp}$ & $4,52 \%$ & 7 \\
\hline Haemophilus sp & $4,52 \%$ & 7 \\
\hline Acinetobacter Iwoffii & $2,58 \%$ & 4 \\
\hline Bacilo gram-positivo & $2,58 \%$ & 4 \\
\hline Enterococcus sp & $1,94 \%$ & 3 \\
\hline Proteus sp & $1,94 \%$ & 3 \\
\hline Streptococcus gama hemolítico & $1,29 \%$ & 2 \\
\hline Serratia $\mathrm{sp}$ & $1,29 \%$ & 2 \\
\hline Citrobacter diversus & $1,29 \%$ & 2 \\
\hline Corynebacterium xerosis & $1,29 \%$ & 2 \\
\hline Propionibacterium acnes & $0,65 \%$ & 1 \\
\hline Mycobacterium abcessus & $0,65 \%$ & 1 \\
\hline Morganella morganii & $0,65 \%$ & 1 \\
\hline Escherichia coli & $0,65 \%$ & 1 \\
\hline Enterobacter sp & $0,65 \%$ & 1 \\
\hline Burckolderia cepacia & $0,65 \%$ & 1 \\
\hline Klebsiella sp & $0,65 \%$ & 1 \\
\hline Hafnia alvei & $0,65 \%$ & 1 \\
\hline
\end{tabular}

\section{DISCUSSÃO}

O tratamento eficaz das endoftalmites inclui a identificação correta e precoce do agente etiológico, através da cultura do humor vítreo e/ou aquoso, para pronta instituição da antibioticoterapia. Em nosso estudo, observamos apenas $29,1 \%$ de positividade nas culturas realizadas. Alguns autores mostraram que, em casos clinicamente suspeitos de endoftalmite, $24,0 \%$ e $54,05 \%$ das culturas foram positivas, respectivamen$\mathrm{te}^{(23-24)}$. Em estudos nacionais, a sensibilidade da cultura para caracterização microbiológica dos casos de endoftalmite foi de $32 \%$ e $85 \%$ dos casos $^{(25-26)}$. A baixa sensibilidade da cultura microbiológica pode ocorrer em decorrência de vários fatores, como pequena quantidade de amostra, seqüestro de microrganismos em superfícies sólidas (lente intra-ocular, fragmentos de lentes, cápsula), que leva à diminuição de células no humor vítreo/aquoso, uso de antimicrobianos antes da coleta do material clínico e a presença de microrganismos fastidiosos como agentes causadores de endoftalmites ${ }^{(24)}$.

A aplicação de técnicas de biologia molecular, como a reação de polimerização em cadeia do DNA (do inglês, PCR), pode aumentar significantemente a sensibilidade de detecção de microrganismos no humor vítreo/aquoso. Um aumento da sensibilidade para $92 \%$ e $100 \%$ pode ser observada utilizando a técnica de $\mathrm{PCR}^{(23-24)}$.

Entre os microrganismos isolados, notamos que os grampositivos foram isolados com maior freqüência $(79,35 \%)$ em relação aos gram-negativos $(20,65 \%)$. Outros autores mostraram que a freqüência de microrganismos gram-positivos pode variar de $63,8 \%$ a $86,3 \%$ e gram-negativos, de $11,8 \%$ a $31,4 \%^{(27-29)}$. Em outros estudos nacionais, os microrganismos gram-positivos foram isolados de endoftalmite com maior frequência em relação aos gram-negativos ${ }^{(25-26)}$.

Outros autores investigaram a distribuição dos microrganismos causadores de endoftalmite (pós-operatória, pós-traumática, endógenas e miscelânea) em um intervalo de 6 anos. Seus dados mostram que os principais microrganismos isolados durante o estudo foram os SCoN $(37,10 \%)$, seguidos por Streptococcus grupo viridans $(12,8 \%)$, S. aureus $(7,7 \%)$ e $P$. acnes $(7 \%)$. Nossos dados são, em parte, semelhantes. Os $\mathrm{SCoN}(41,94 \%)$ foram os principais microrganismos isolados, seguidos também por Streptococcus grupo viridans $(14,19 \%)$ e $S$. aureus (8,39\%). Entretanto, houve apenas $0,65 \%$ de isolamento de $P$. acnes, em contraste com $7 \%$. Em contrapartida, $S$. pneumoniae apareceram com uma freqüência de $7,10 \%$, enquanto o trabalho referido revelou $2,9 \%{ }^{(28)}$.

Todos os microrganismos gram-positivos isolados foram sensíveis à vancomicina, estando de acordo com os dados da literatura, que também mostram $100 \%$ de sensibilidade à vancomicina dos microrganismos gram-positivos isolados de endoftalmite $^{(25-28)}$. Em nosso trabalho, obtivemos $100 \%$ de sensibilidade à ciprofloxacina e levofloxacina em microrganismos gram-negativos (com exceção de $P$. aeruginosa, que apresentou sensibilidade diminuída à ciprofloxacina $(83,33 \%)$, e uma eficiência de cobertura menor da ciprofloxacina e da levofloxacina para os microrganismos gram-positivos. As fluoroquinolonas, como ciprofloxacina, ofloxacina e levofloxacina, possuem excelente cobertura para gram-negativos, mas são menos eficazes contra microrganismos gram-positivos, particularmente para Staphylococcus spp e Streptococcus spp $^{(30)}$. 
Tabela 2. Distribuição dos microrganismos isolados em relação à via de aquisição

\begin{tabular}{|c|c|c|c|c|}
\hline \multirow[t]{2}{*}{ Microrganismos isolados $(n=155)$} & \multicolumn{4}{|c|}{ Vias de aquisição } \\
\hline & Pós-operatória & Pós-traumática & Endógena & Desconhecida \\
\hline Streptococcus grupo viridans & 15 & 5 & & 2 \\
\hline Streptococcus pneumoniae & 4 & 4 & & 3 \\
\hline Pseudomonas sp & 4 & 1 & & 2 \\
\hline Haemophilus sp & 1 & 3 & & 3 \\
\hline Enterococcus sp & 3 & & & \\
\hline Proteus sp & 2 & 1 & & \\
\hline Streptococcus gama hemolítico & 1 & & & 1 \\
\hline Serratia sp & 1 & 1 & & \\
\hline Citrobacter diversus & 1 & 1 & & \\
\hline Escherichia coli & & & 1 & \\
\hline Enterobacter sp & & & & 1 \\
\hline Burckolderia cepacia & 1 & & & \\
\hline Klebsiella sp & & 1 & & \\
\hline Hafnia alvei & 1 & & & \\
\hline Total & 94 & 25 & 4 & 32 \\
\hline
\end{tabular}

\begin{tabular}{|c|c|c|c|c|c|c|c|c|c|c|c|}
\hline \multirow[t]{2}{*}{$\begin{array}{l}\text { Microrganismo } \\
\text { sem } \\
\text { antibióticos }\end{array}$} & \multicolumn{2}{|c|}{$\begin{array}{l}\text { Staphylo- } \\
\text { coccus } \\
\text { coagulase } \\
\text { negativa }\end{array}$} & \multicolumn{2}{|c|}{$\begin{array}{l}\text { Strepto- } \\
\text { coccus } \\
\text { grupo } \\
\text { viridans }\end{array}$} & $\begin{array}{c}\text { Strepto- } \\
\text { coccus } \\
\text { pneumoniae }\end{array}$ & \multicolumn{2}{|c|}{$\begin{array}{l}\text { Staphylo- } \\
\text { coccus } \\
\text { aureus }\end{array}$} & $\begin{array}{c}\text { Pseudomonas } \\
\text { sp }\end{array}$ & $\begin{array}{c}\text { Haemophilus } \\
\text { sp }\end{array}$ & $\begin{array}{c}\text { Enterococcus } \\
\text { sp }\end{array}$ & \multirow[t]{2}{*}{$\begin{array}{c}\text { Outros } \\
\text { bacilos } \\
\text { Gram- } \\
\text { negativos } \\
\text { n (\%) }\end{array}$} \\
\hline & $\mathrm{n}$ & (\%) & $\mathrm{n}$ & $(\%)$ & $(\%)$ & $\mathbf{n}$ & $(\%)$ & n $\quad(\%)$ & n $\quad(\%)$ & $(\%)$ & \\
\hline Amicacina & - & & - & & - & - & & $7 \quad(85,71)$ & $7(71,43)$ & - & $17(94,12)$ \\
\hline Tobramicina & - & & - & & - & - & & $7 \quad(57,14)$ & $7(85,71)$ & - & $17(88,24)$ \\
\hline Cefazolina & 42 & $(97,62)$ & 14 & $(85,71)$ & - & 7 & $(100,0)$ & - & $5(80,00)$ & - & $10(60,00)$ \\
\hline Ceftazidima & 42 & $(76,19)$ & 14 & $(78,57)$ & - & 7 & $(100,0)$ & $4(100,0)$ & $5(100,0)$ & - & $11(72,73)$ \\
\hline Gatifloxacina & 36 & $(100,0)$ & 12 & $(75,00)$ & $5(80,00)$ & 7 & $(100,0)$ & $3(100,0)$ & $4(100,0)$ & $2(100,0)$ & $6(100,0)$ \\
\hline Ciprofloxacina & 65 & $(92,31)$ & 22 & $(45,45)$ & $11(54,55)$ & 12 & $(83,33)$ & $6(83,33)$ & $7(100,0)$ & $3(33,33)$ & $17(100,0)$ \\
\hline Levofloxacina & 20 & $(95,00)$ & 6 & $(50,00)$ & $3(100,0)$ & 1 & $(100,0)$ & $1(100,0)$ & $2(100,0)$ & $2(100,0)$ & $5(100,0)$ \\
\hline Moxifloxacina & 36 & $(100,0)$ & 12 & $(91,67)$ & $5(100,0)$ & 7 & $(100,0)$ & $3(100,0)$ & $4(100,0)$ & $3(100,0)$ & $6(100,0)$ \\
\hline Eritromicina & 45 & $(66,67)$ & 16 & $(81,25)$ & $6(100,0)$ & 7 & $(71,43)$ & - & - & $2(50,00)$ & $15(26,67)$ \\
\hline Gentamicina & 64 & $(82,81)$ & - & & - & 13 & $(84,62)$ & $7 \quad(57,14)$ & - & $2(100,0)^{\star}$ & $17(82,35)$ \\
\hline Vancomicina & 65 & $(100,0)$ & 22 & $(100,0)$ & $11(100,0)$ & 13 & $(100,0)$ & - & - & $3(100,0)$ & - \\
\hline Cloranfenicol & 64 & $(87,50)$ & 22 & $(100,0)$ & $11(100,0)$ & 13 & $(84,62)$ & $7 \quad(14,29)$ & $7(85,71)$ & $3(66,67)$ & $17(70,59)$ \\
\hline Oxacilina & 65 & $(83,08)$ & - & & $11(72,73)$ & 13 & $(84,62)$ & - & - & - & - \\
\hline Penicilina & 59 & $(18,64)$ & 17 & $(76,47)$ & $8(100,0)$ & 12 & $(33,33)$ & - & $6(66,67)$ & $2(100,0)$ & - \\
\hline
\end{tabular}

Notamos maior atividade da levofloxacina em relação à ciprofloxacina para microrganismos gram-positivos, como mostrado também por outros autores ${ }^{(28,30-31)}$. Para os estafilococos $(S$. aureus e SCoN), a sensibilidade à levofloxacina foi de $95,24 \%$ e à ciprofloxacina, de $89,61 \%$. Streptococcus sp mostraram uma porcentagem de sensibilidade à levofloxacina e ciproflo- xacina de $66,7 \%$ e $48,5 \%$ respectivamente. O uso rotineiro em larga escala da ciprofloxacina na prática clínica tanto para terapêutica como profilaxia, pode ser responsável pelo aumento de microrganismos resistentes a esse agente. Apesar de não apresentar cobertura completa para todos os microrganismos gram-positivos, como mostram nossos dados e de outros au- 
tores $^{(28,30-31)}$, após administração oral, a levofloxacina atinge, nos humores aquoso e vítreo, concentrações acima das concentrações inibitórias mínimas contra um grande número de microrganismos causadores de endoftalmite, e pode servir como adjuvante na antibioticoterapia desta infecção ${ }^{(32)}$.

Dois novos membros da classe das fluoroquinolonas, gatifloxacina e moxifloxacina, podem suprir as falhas na cobertura antimicrobiana das outras fluoroquinolonas. Gatifloxacina e moxifloxacina possuem amplo espectro de ação contra os principais patógenos oculares, tendo demonstrado um alto grau de atividade in vitro contra microrganismos gram-positivos como S. aureus, SCoN, Streptococcus grupo viridans e outros microrganismos gram-positivos ${ }^{(33-35)}$. Tanto gatifloxacina como moxifloxacina apresentam boa penetração no humor aquoso e conseguem atingir ou exceder a concentração inibitória mínima para os principais patógenos bacterianos após administração oral, e devem ser preferidas, ao invés das outras fluoroquinolonas, para antibioticoterapia de endoftalmites bacterianas $^{(36)}$. Entretanto, isolados bacterianos oriundos de endoftalmite já revelam resistência a essas novas fluoroquinolonas. Em um estudo que avaliou o uso profilático de fluoroquinolonas e o perfil de sensibilidade bacteriana de microrganismos isolados de casos de endoftalmite aguda pós-operatória 31 pacientes, dos 42 que desenvolveram endoftalmite, fizeram uso profilático de gatifloxacina ou moxifloxacina. O perfil de sensibilidade antibiótica foi avaliado para 14 microrganismos gram-positivos e a sensibilidade para gatifloxacina e moxifloxacina foi de $38 \%{ }^{(30)}$. Outro estudo recente também demonstrou relativo aumento de resistência in vitro à fluoroquinolonas em Staphylococcus aureus meticilina-resistentes (MRSA) isolados de infecções oculares ${ }^{(37)}$. Em nosso estudo, as amostras avaliadas até o ano de 2005 apresentaram 100\% de sensibilidade à gatifloxacina e moxifloxacina. Entre os Staphylococcus sp resistentes à oxacilina (13/78), 100\% foram sensíveis à gatifloxacina e moxifloxacina, e apresentaram sensibilidade diminuída apenas para ciprofloxacina $(53,85 \%)$, amicacina $(53,85 \%)$ e tobramicina $(38,46 \%)$. No entanto, os dados de sensibilidade aos antimicrobianos dos SCoN isolados de endoftalmite pelo Laboratório de Microbiologia Ocular (LOFT) do Departamento de Oftalmologia da UNIFESP no ano de 2006 mostram uma tendência à diminuição da sensibilidade para essas duas fluoroquinolonas. Desses SCoN, 35,7\% apresentaram sensibilidade diminuída à gatifloxacina e moxifloxacina ${ }^{(38)}$. Outra avaliação da sensibilidade às fluoroquinolonas em amostras de SCoN isoladas pelo LOFT mostrou que tanto moxifloxacina como gatifloxacina apresentaram maior potência contra os SCoN sensíveis à oxacilina $\left(\mathrm{CIM}_{90} ; 0,125\right.$ 1/4g/ml para ambas as drogas) quando comparada à potência contra os SCoN resis-

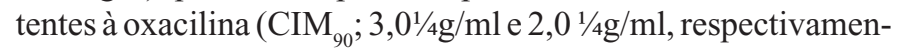
te). Considerando os valores de CIM obtidos nesse estudo, $95,5 \%$ dos $\mathrm{SCoN}$ sensíveis à oxacilina apresentaram sensibilidade à moxifloxacina e gatifloxacina, enquanto apenas 33,33\% e $38,10 \%$ dos $\mathrm{SCoN}$ resistentes à oxacilina foram sensíveis às duas fluoroquinolonas, respectivamente. No entanto, esse estudo incluiu amostras de $\mathrm{SCoN}$ isoladas de casos de ceratite, conjuntivite e endoftalmite e a avaliação da sensibilidade não foi estratificada por sítio de infecção ${ }^{(39)}$. Avaliação do perfil de sensibilidade à fluoroquinolonas em $\mathrm{SCoN}$ isolados de pacientes com endoftalmite pós-operatória atendidos entre 1990 e 2004 pelo Bascom Palmer Eye Institute, mostra que a porcentagem de sensibilidade desses isolados à gatifloxacina e moxifloxacina foi de $96,6 \%$ de 1990 a 1994, 78,2\% de 1995 a 1999, e 65,4\% de 2000 a $2004^{(31)}$. Entre os SCoN com sensibilidade diminuída a essas fluoroquinolonas isolados em 2006 pelo LOFT, 80,0\% foram resistentes à meticilina ${ }^{(38)}$.

Aumento da resistência à gatifloxacina e moxifloxacina é relatado também entre cepas de Staphylococcus aureus meticilina-resistentes (MRSA) isolados tanto de infecções oculares como em infecções não oculares. Foi demonstrado que entre os MRSA isolados de endoftalmite avaliados quanto à sensibilidade às fluoroquinolonas, 71,0\% apresentaram resistentes à gatifloxacina e 68,0\% à moxifloxacina. Além disso, entre os MRSA isolados de outros sítios infecciosos por esse mesmo hospital, no ano de $2005,87,0 \%$ e $82,0 \%$ dos isolados foram resistentes à gatifloxacina e moxifloxacina, respectivamente ${ }^{(37)}$.

\section{CONCLUSÃO}

Os dados apresentados revelam a necessidade da utilização de técnicas mais sensíveis na detecção de microrganismos causadores de endoftalmite, incluindo metodologia molecular. Uma terapia antimicrobiana ou profilaxia empírica deve ser ativa contra os microrganismos gram-positivos particularmente contra estafilococos. Tendências de resistência bacteriana aos antimicrobianos utilizados em oftalmologia podem ser detectadas em estudos de vigilância microbiológica, propiciando uma adequação rápida de esquemas terapêuticos ou profiláticos.

\section{ABSTRACT}

Purpose: To assess the distribution of microorganisms isolated from patients with bacterial endophthalmitis and their antimicrobial susceptibility. Methods: Retrospective analysis of medical and microbiological records of patients with suspected diagnosis of endophthalmitis and bacterial culture-proven at the Department of Ophthalmology, UNIFESP, between January 12000 and December 31 2005. Results: 153 (33.9\%) of 451 patients showed positive bacterial culture. A total of 155 microorganisms were isolated, $79.35 \%$ were gram-positive and $20.65 \%$ gram-negative. Staphylococcus (CoNS) (41.94\%) were the most frequently isolated. The antimicrobial susceptibility for gram-negative microorganisms was as follows: amikacin $87.10 \%$, tobramycin $80.65 \%$, ciprofloxacin $96.67 \%$, levofloxacin, gatifloxacin and moxifloxacin $100 \%$, ceftazidime $85.0 \%$, and gentamicin $80.65 \%$. Vancomycin sensitivity among grampositive microorganisms was $100 \%$. S. aureus and CoNS showed $83.33 \%$ of susceptibility to oxacillin, $89.61 \%$ to 
ciprofloxacin and $100 \%$ to gatifloxacin and moxifloxacin. The main acquisition mechanism was postoperative $(60.65 \%)$. Conclusion: We detected a low sensitivity of vitreous/aqueous culture for the etiologic diagnosis of endophthalmitis. The empiric antimicrobial therapy or prophylaxis should be active against gram-positive bacteria, particularly staphylococci. Surveillance studies of bacterial resistance are important for a better utilization of antimicrobials in this clinical setting.

Keywords: Eye infections, bacterial; Endophthalmitis/etiology; Endophthalmitis/surgery; Aqueous humor/microbiology; Drug resistance, microbial; Microbial sensitivity tests

\section{REFERÊNCIAS}

1. Jackson TL, Eykyn SJ, Graham EM, Stanford MR. Endogenous bacterial endophthalmitis: a 17-year prospective series and review of 267 reported cases. Surv Ophthalmol. 2003;48(4):403-23.

2. Callegan MC, Engelbert M, Parke DW 2nd, Jett BD, Gilmore MS. Bacterial endophthalmitis: epidemiology, therapeutics, and bacterium-host interactions. Clin Microbiol Rev. 2002;15(1):111-24.

3. Taban M, Behrens A, Newcomb RL, Nobe MY, McDonnell PJ. Incidence of acute endophthalmitis following penetrating keratoplasty: a systematic review. Arch Ophthalmol. 2005;123(5):605-9.

4. Han DP, Wisniewski SR, Wilson LA, Barza M, Vine AK, Doft BH, et al. Spectrum and susceptibilities of microbiologic isolates in the Endophthalmitis Vitrectomy Study. Am J Ophthalmol. 1996;122(1):1-17. Erratum in: Am J Ophthalmol. 1996;122(6):920

5. Kresloff MS, Castellarin AA, Zarbin MA. Endophthalmitis. Surv Ophthalmol. 1998;43(3):193-224.

6. West ES, Behrens A, McDonnell PJ, Tielsch JM, Schein OD. The incidence of endophthalmitis after cataract surgery among the U.S. Medicare population increased between 1994 and 2001. Ophthalmology. 2005;112(8):1388-94.

7. Read RW. Endophthalmitis. In: Yanoff M, Duker JS, editors. Ophthalmology. St Louis, MO: Mosby; 2004. p.1152-7.

8. Aaberg TM Jr, Flynn HW Jr, Schiffman J, Newton J. Nosocomial acute-onset postoperative endophthalmitis survey: a 10-year review of incidence and outcomes. Ophthalmology. 1998;105(6):1004-10.

9. Samson CM, Foster CS. Chronic postoperative endophthalmitis. Int Ophthalmol Clin. 2000;40(1):57-67.

10. Speaker MG, Milch FA, Shah MK, Eisner W, Kreiswirth BN. Role of external bacterial flora in the pathogenesis of acute postoperative endophthalmitis. Ophtalmology. 1991;98(5):639-49; discussion 650 .

11. O'Brien TP, Choi S. Trauma-related ocular infections. Int Ophthalmol Clin N Am. 1995;8:667-79.

12. Thompson ST, Parver LM, Enger CL, Meiler WF, Liggett PE. Infectious endophthalmitis after penetrating injuries with retained intraocular foreign bodies. Ophthalmology. 1993;100(10):1468-74.

13. Shrader SK, Band JD, Lauter CB, Murphy P. The clinical spectrum of endophthalmitis: incidence, predisposing factors, and features influencing outcome. J Infect Dis. 1990;162(1):115-20.

14. Margo CE, Mames RN, Guy JR. Endogenous Klebsiella endophthalmitis. Report of two cases and review of the literature. Ophthalmology. 1994;101(7):1298-301.

15. Romero CF, Rai MK, Lowder CY, Adal KA. Endogenous endophthalmitis: case report and brief review. Am Fam Physician. 1999;60(2):510-4.

16. Tseng CY, Liu PY, Shi ZY, Lau YJ, Hu BS, Shyr JM, et al. Endogenous endophthalmitis due to Escherichia coli: case report and review. Clin Infect Dis. 1996;22(6):1107-08

17. Lauer AK, Riley K, Wentzien J, Marsal SW. Acute painful vision loss and acute abdomen: a case of endogenous Clostridium perfringens endophthalmitis. Can J Ophthalmol. 2005;40(2):208-10.

18. Garg SJ, Nelson M, Kanitkar K, Kleiner R. Subretinal abscess and endogenous endophthalmitis caused by beta-lactamase-positive Moraxella species. Retina. 2006;26(8):968-9.

19. Gorroño-Echebarria MB, Rojo G. [Endogenous endophthalmitis caused by Streptococcus pneumoniae in an immune competent patient]. Med Clin (Barc). 2006;127(9):357-8. Spanish.

20. Wu Z, Uzcategui N, Chung M, Song J, Lim JI. Group B streptococcal endogenous endophthalmitis in a neonate. Retina. 2006;26(4):472-3.

21. Baquero Latorre H, Neira SF, Gonzales Vargas T. [Endogenous endophthalmitis due to Serratia marcescens in the course of early neonatal sepsis]. An Pediatr (Barc). 2006;64(3):291-2.

22. Koneman EW, Allen SD, Janda WM, Schreckenberger PC, Winn Jr WC. Diagnóstico microbiológico: texto e atlas colorido. $5^{\underline{a}}$ ed. Rio de Janeiro: MEDSI; 2001.

23. Lohmann CP, Linde HJ, Reischl U. Improved detection of microorganisms by polymerase chain reaction in delayed endophthalmitis after cataract surgery. Ophthalmology. 2000;107(6):1047-51; discussion 1051-2.

24. Okhravi N, Adamson P, Carroll N, Dunlop A, Matheson MM, Towler HM, et al. PCR-based evidence of bacterial involvement in eyes with suspected intraocular infection. Invest Ophthalmol Vis Sci. 2000;41(11):3474-9.

25. Uesugui E, Cypel-Gomes MC, Atique D, Goulart DG, Gallucci FR, Nishiwaki-Dantas MC, et al. Identificação laboratorial dos patógenos oculares mais freqüentes e sua suscetibilidade in vitro aos agentes antimicrobianos. Arq Bras Oftalmol. 2002;65(3):339-42.

26. Schirmbeck T, Romão E, Rodrigues ML, Figueiredo JF. Endoftalmite: Uma análise de 58 casos. Arq Bras Oftalmol. 2000;63(1):39-44.

27. Ng JQ, Morlet N, Pearman JW, Constable IJ, McAllister IL, Kennedy CJ, Isaacs T, Semmens JB; Team EPSWA. Management and outcomes of postoperative endophthalmitis since the endophthalmitis vitrectomy study: the Endophthalmitis Population Study of Western Australia (EPSWA)'s fifth report. Ophthalmology. 2005;112(7):1199-206. Comment in: Ophthalmology. 2006;113(8):1472-3; author reply 1473.e1-2.

28. Benz MS, Scott IU, Flynn HW Jr, Unonius N, Miller D. Endophthalmitis isolates and antibiotic sensitivities: a 6-year review of culture-proven cases. Am J Ophtahlmol. 2004;137(1):38-42. Comment in: Am J Ophthalmol. 2004;137(6): 1167-8; author reply 1168; Am J Ophthalmol. 2004;137(6):1169; author reply 1169-70.

29. Kunimoto DY, Das T, Sharma S, Jalali S, Majji AB, Gopinathan U, et al. Microbiologic spectrum and susceptibility of isolates: part I. Postoperative endophthalmitis. Endophthalmitis Research Group. Am J Ophthalmol. 1999;128(2): 240-2.

30. Deramo VA, Lai JC, Fastenberg DM, Udell IJ. Acute endophthalmitis in eyes treated prophylactically with gatifloxacin and moxifloxacin. Am J Ophthalmol. 2006;142(5):721-5.

31. Miller D, Flynn PM, Scott IU, Alfonso EC, Flynn HW Jr. In vitro fluoroquinolone resistance in staphylococcal endophthalmitis isolates. Arch Ophthalmol. 2006;124(4):479-83.

32. Fiscella RG, Nguyen TK, Cwik MJ, Phillpotts BA, Friedlander SM, Alter $\mathrm{DC}$, et al. Aqueous and vitreous penetration of levofloxacin after oral administration. Ophthalmology. 1999;106(12):2286-90.

33. Mather R, Karenchak LM, Romanowski EG, Kowalski RP. Fourth generation fluoroquinolones: new weapons in the arsenal of ophthalmic antibiotics. Am J Ophthalmol. 2002;133(4):463-6.

34. Mah FS. Fourth-generation fluoroquinolones: new topical agents in the war on ocular bacterial infections. Curr Opin Ophthalmol. 2004;15(4):316-20.

35. Hwang DG. Fluoroquinolone resistance in ophthalmology and the potential role for newer ophthalmic fluoroquinolone. Surv Ophthalmol. 2004;49(2):S79-S83.

36. Hariprasad SM, Shah GK, Mieler WF, Feiner L, Blinder KJ, Holekamp NM, et al. Vitreous and aqueous penetration of orally administered moxifloxacin in humans. Arch Ophthalmol. 2006;124(2):178-82.

37. Kotlus BS, Wymbs RA, Vellozzi EM, Udell IJ. In vitro activity of fluoroquinolones, vancomycin, and gentamicin against methicillin-resistant Staphylococcus aureus ocular isolates. Am J Ophthalmol. 2006;142(5):726-9.

38. Bispo PJ, Melo GB, Yu MCZ, Martino MD, Höfling-Lima AL, Pignatari AC. Distribuição das espécies e perfil de sensibilidade a quinolonas de Staphylococcus coagulase-negativa (SCN) isolados de endoftalmite [CD-ROM]. In: XXIV Congresso Brasileiro de Microbiologia; 2007 Out 2-6, Brasília, Brasil.

39. Oliveira AD, Höfling-Lima AL, Belfort R Jr, Gayoso MF, Francisco W. Fluoroquinolone susceptibilities to methicillin-resistant and susceptible coagulase-negative Staphylococcus isolated from eye infection. Arq Bras Oftalmol. 2007;70(2):286-9. 